\title{
Analysis of Post-Disaster Construction Safety Management: Challenges Faced by Mozambican Government due to Natural Disasters (Cyclone, Floods)
}

\author{
Stella Tsambe ${ }^{1}$, Kim, Dae Young* \\ ${ }^{1}$ Department of Architectural Engineering, Pusan National University, South Korea \\ * Department of Architectural Engineering, Pusan National University, South Korea
}

\begin{abstract}
:
Due to its geographical location and climate Mozambique has constantly been affected by floods and storms over the past decade. The lack of fast response often causes the communities to build back using debris. This form of reconstruction is counterproductive to mitigation and is a risk to safety. According to the Mozambican Contingency Plan for 2019, Mozambique remains at threat of difficulty in recovery due to its weak implementation of measures for disaster risk reduction in activities centered around resettlement and reconstruction. The goal of this study is to identify the challenges faced by the Mozambican government in Construction Management in Post disaster situation where the goal is to rebuild safer. Through analyzing data released from surveys and questionnaires conducted by both governmental and non-governmental institutions 79 tasks related to post disaster construction management were identified and classified in the categories of: design, government structure, laws and policies, technical assistance, communication and community involvement. These tasks were then analyzed through an IPA graph in order to evaluate their current impact. The findings of this study are country specific but are paired and compared with findings from other authors who have developed similar studies in various underdeveloped countries. It is hoped that this study will provide basis for future research and development strategies in the area of Post Disaster Construction Safety Management and help to identify solutions for the many challenges faced by the existing systems.
\end{abstract}

Keywords: Construction Management, safety, post disaster reconstruction, challenges 